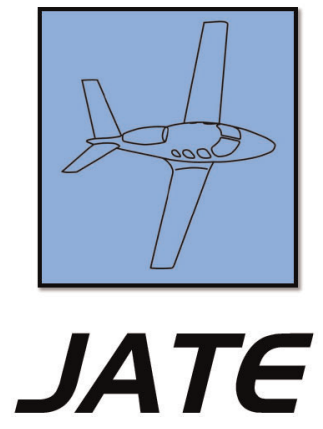

Journal of Aviation Technology and Engineering 7:2 (2018) 57-66

\title{
Pilot Visual Detection of Small Unmanned Aircraft Systems (sUAS) Equipped with Strobe Lighting
}

\author{
Ryan J. Wallace \\ Embry-Riddle Aeronautical University
}

\begin{abstract}
Jon M. Loffi, Samuel M. Vance, Jamey Jacob, Jared C. Dunlap, and Taylor A. Mitchell
Oklahoma State University
\end{abstract}

\begin{abstract}
When operating under Visual Flight Rules, pilots primarily rely on visual scanning to avoid other aircraft and airborne collision threats. Records from the Federal Aviation Administration indicate that near encounters with unmanned aircraft are on the rise, reaching 1,761 reported unmanned aircraft system (UAS) sightings or near-misses in 2016. This study sought to assess the effectiveness of pilot visual detection of UAS platforms that were equipped with strobe lighting. A sample of 10 pilots flew a general aviation aircraft on a scripted series of five intercepts with a small UAS (sUAS) that was equipped with strobe lighting. Participants were asked to indicate when they visually detected the unmanned aircraft. Geolocation information for both the aircraft and sUAS platform was compared to assess visibility distance. Findings were used to evaluate the efficacy of daytime strobe lighting as a method to enhance pilot sUAS detection, visibility, and collision avoidance. Participants detected the unmanned aircraft during 7.7\% of the intercepts. Due to a lack of data points, the authors were unable to conclusively determine if strobe lighting improved UAS visual detection. The authors recommend further research to explore the effectiveness of using sUAS-mounted strobe lights for nighttime visual detection.
\end{abstract}

Keywords: sUAS, unmanned aircraft system, mid-air collision avoidance (MACA), strobe light, high-intensity xenon lighting, aircraft lighting

\section{Introduction}

The Federal Aviation Administration (FAA) relies on both unmanned aircraft operators and manned aircraft pilots to use see-and-avoid practices to avoid collisions. See-and-avoid procedures include a myriad of recommendations which include understanding human vision limitations, practicing recommended visual scanning techniques, preflight planning, performing clearing maneuvers, adhering to airspace restrictions and airport traffic patterns, employing effective communication, utilizing collision-avoidance technology, making use of available ATC traffic advisories and safety pilots (FAA, 2016).

Codified in 14 CFR Part 91.113(b), the FAA highlights the need for continued pilot vigilance to see and avoid other aircraft, as well as follow established right of way rules (General Operating Rules, 2017). The FAA also charges

All correspondence concerning this article should be directed to Ryan J. Wallace at ryan.wallace@erau.edu. 


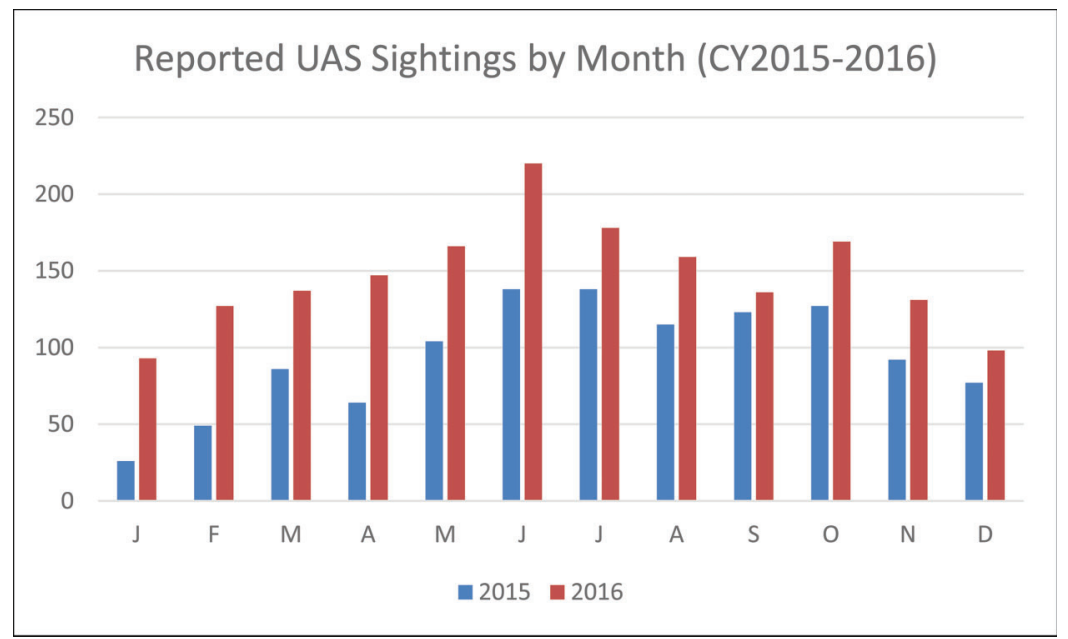

Figure 1. Reported UAS sightings by month (2015-2016). Derived from FAA UAS sightings reports obtained from https://www.faa.gov/uas/resources/uas_sightings_report/.

commercial unmanned aircraft system (UAS) operators flying under 14 CFR 107.31(a) with practicing see-and-avoid procedures, which include requirements for maintaining visual contact throughout the UAS flight and observing for aerial traffic and hazards (Small Unmanned Aircraft Systems, 2017). The agency gives similar guidance to model aircraft and hobbyist UAS operators in 14 CFR 101.41(d), requiring that operators conduct flights "in a manner that does not interfere and gives way to any manned aircraft" (Moored Balloons, Kites, Amateur Rockets, Unmanned Free Balloons, and Certain Model Aircraft, 2017).

\section{Problem}

Unfortunately, data trends may suggest that see-andavoid strategies are not adequately working to stave off aircraft-UAS near misses. Close encounters between manned aircraft and UAS continue to remain problematic for the FAA, as evidence suggests that pilot close encounters with UAS platforms are continuing to increase. A FAA database revealed more than 1,761 sightings or near encounters were reported in 2016 - an average of nearly 150 a month (FAA, 2017). This represents an increase of 622 reported incidents over 2015 sightings, which only numbered 1,139 (FAA, 2017). Figure 1 shows a year-on-year comparison of UAS encounter data plotted in monthly increments.

\section{History of Close Encounters}

\section{Gettinger and Michel study}

Gettinger and Michel (2015) assessed 921 UAS encounter incidents that occurred between December 2013 and September 2015. Their study clarified that while UAS sightings were numerous, not all UAS sightings presented a genuine risk of collision (Gettinger \& Michel, 2015). The researchers categorized UAS encounter incidents as "close encounters" only if aircraft-UAS proximity was less than 500 feet, the pilot declared a near mid-air collision (NMAC) or used other similar language, or the aircraft initiated evasive action; otherwise, encounters were deemed "sightings" (Gettinger \& Michel, 2015, p. 5). Gettinger and Michel (2015) determined that that the vast majority- $64.5 \%$ of reported UAS encounters-were considered relatively benign sightings, whereas only $35.5 \%$ of UAS encounters were close encounters and presented a genuine collision risk. It is notable to highlight that in $21.2 \%(n=51)$ of reported UAS close encounters, aircraft passed within 50 feet or less-effectively a NMAC. In $8.6 \%$ of close encounters $(n=28)$, pilots initiated evasive action. The Gettinger and Michel study also determined that $90.2 \%$ of UAS encounters occurred at altitudes above 400 feet, where most UAS operations are prohibited. Additionally, the researchers identified that UAS encounters were occurring at disproportionally higher rates around major airports (Gettinger \& Michel, 2015). Michel and Gettinger (2016) updated their assessment following an FAA data release that included 582 UAS encounter incidents that occurred from August 2015 to January 2016. While the rate of encounters grew, the overall findings remained largely consistent with the original study (Michel \& Gettinger, 2016).

\section{Academy of Model Aeronautics study}

In a series of reports using FAA UAS sightings data collected from November 2014 to September 2016, the Academy of Model Aeronautics (AMA) identified key findings related to UAS near encounters. The organization assessed 2,616 reports of UAS sightings, encounters, or near-misses divided into three datasets by date of FAA data release (AMA, 2016; AMA, 2017). The three FAA reporting periods included November 2014 to August 2015; August 2015 to January 2016; and, February 2016 to September 2016. Diverging from the methodology used by the Gettinger and Michel (2015) study, the AMA taxonomy method only categorized a report as a NMAC if the report 
text contained an "explicit notation of 'NMAC' or near miss" (AMA, 2017, p. 2). The AMA (2016) asserted that each of the respective datasets reflected a NMAC rate much lower than the Gettinger and Michel (2015) findings, at $3.5 \%(n=27, N=764), 3.3 \%(n=19, N=582)$, and $3.4 \%(n=44, N=1,270)$. The AMA further pointed out that among the three datasets, pilots only took evasive action during $1.3 \%, 2.4 \%$, and $2.9 \%$ of total reports (AMA, 2017). The AMA further highlighted discrepancies in the FAA's sighting reports, which indicated that while a UAS may have been sighted by an aircraft, it was likely that several of those sightings involved UAS operations that were in compliance with existing FAA UAS regulations. Finally, the AMA criticized the validity of FAA data, identifying several data points that included sightings of balloons, birds, kites, parasails, or other unidentified objects (AMA, 2017).

\section{Literature Review}

As of October 2017, few studies have been conducted to evaluate the effectiveness of the FAA's see-and-avoid concept with respect to unmanned aircraft.

\section{UAS Visibility Studies}

\section{Ohio University study}

Kephart and Braasch (2008) conducted a series of visibility experiments to compare the effectiveness of UAS operator and pilot detection of obstacles and conflicting air traffic. While their overarching purpose was to test the efficacy of a forward-looking camera system, their study provided an initial basis for pilot traffic detection visibility thresholds. During the test, seven experimental participants flew a Piper Saratoga, which was intercepted twice by an unmanned aircraft simulated by a Piper Warrior aircraft. Participants detected the intruding aircraft during $92.9 \%$ of the intercepts $(n=13, N=14)$ with detection ranges varying from a minimum of $0.309 \mathrm{SM}$ to a maximum of 2.336 SM. The mean detection distance for the experiment was $1.511 \mathrm{SM}$ for the $90^{\circ}$ intercept condition and 1.038 SM for the head-on intercept condition. Kephart and Braasch (2008) explained their findings fell generally within the estimated detection range of 1.14 to $2.3 \mathrm{SM}$, based on the Air Force Research Lab Operational Encounter Model (Kochenderfer, Espindle, Kucher, \& Griffith, 2008). While the study provides a reasonable baseline for pilot detection, the use of a civil aircraft in lieu of a UAS potentially compromises the validity of its findings. Ultimately, the size and cross section of the intruding Piper Warrior aircraft are substantially larger than those of most UAS in the current marketplace, making these findings largely invalid.

\section{Colorado Agricultural Aviation Association study}

In 2015, the Colorado Agricultural Aviation Association conducted an experiment to assess pilot visibility of unmanned aircraft during aerial application operations (Maddocks $\&$ Griffitt, 2015). Four fixed-wing agricultural aircraft and one helicopter conducted brief clearing maneuvers over five rural fields. One field was devoid of UAS activity, UAS activity was being actively conducted in two fields, and two additional fields showed evidence of UAS activity via the presence of high-visibility tarps marked with "UAV." Participants were not informed of the presence or absence of UAS activity in each test field. Of the four participating fixedwing aircraft, only one reported briefly glimpsing a UAS in flight - a $12.5 \%$ overall detection rate; whereas, the participating helicopter reported seeing both UAS aircraft-a 100\% detection rate. Among those successful detections, both fixedwing and helicopter crews quickly lost visual contact with the UAS and were unable to track the platform after initial detection. All pilots reported sighting the high-visibility ground markings. In their report, Maddocks and Griffitt (2015) recommended further study of visibility enhancements for UAS, including: external UAS lighting, mirrors, coloration, transponders, and other technological solutions.

\section{Oklahoma State University study}

Loffi, Wallace, Jacob, and Dunlap (2016) conducted a subsequent visibility study based in large part on the findings and methodology of both the Kephart and Braasch (2008) and Maddocks and Griffitt (2015) studies. During the experiment, 20 pilot-qualified participants each flew a Cessna 172 aircraft on a series of six scripted intercepts with small UAS (sUAS) to determine the mean detection distance of a sUAS for an aware pilot. The experiment utilized an Iris quadcopter sUAS and Anaconda fixed-wing sUAS, both of which were colored white to enhance reflection and visibility. Participants detected the Iris quadcopter sUAS during $36.8 \%(n=28)$ intercepts and the Anaconda fixed-wing sUAS during $87.0 \%(n=20)$ of the intercepts. The mean detection range of the Iris was less than 0.10 SM; and the mean detection range of the Anaconda was 0.49 SM. The researchers further identified that the participants were notably poor at accurately estimating distance to sighted sUAS. Participants generally overestimated the range to the smaller Iris sUAS by $0.20 \mathrm{SM}$, and underestimated the range to the larger Anaconda by 0.25 SM. The researchers identified several likely human factor conditions that adversely affected both pilot visibility and estimation judgement to the sUAS. Researchers recommended follow-on studies to examine UAS lighting and night operations, markings and color contrast, and additional testing of transponder systems. The research concluded with several operational suggestions to improve sUAS detection and avoidance.

\section{Aircraft Lighting Research}

As of October 2017, several studies have been conducted to evaluate the effectiveness of various forms of lighting to improve aircraft visibility during both daytime and nighttime conditions. 


\section{Exterior lighting study}

In a study for the FAA, the Applied Psychology Corporation published a report detailing the effectiveness of various forms of exterior aircraft lighting in preventing mid-air collisions (Projector, 1962). The expansive report identified various implementations of navigational lighting, including a thorough evaluation of color schemes and flash patterns to assess aircraft detectability during daytime, nighttime, and reduced visibility conditions. Projector (1962) observed that visibility results varied considerably among and between individuals. In clear skies at night, the visibility of a 100-candle light exceeded 10 miles (Projector, 1962). Projector (1962) further identified that "the flashing of a light signal at intervals makes more efficient use of luminous energy in producing intensity (and therefore visibility) than distributing the same energy in a steady-burning light" (p. 36). While flashing improved initial detectability, Bullough (2011) suggests that it hindered recognition of a collision: "Steady burning [light] sources provide superior guidance regarding closure detection than flashing lights" (p. 18).

Daytime tests revealed much poorer performance of aircraft lighting. According to Projector (1962), "extremely high intensities would be required to provide adequate signal visibilities throughout daylight conditions" (p. 53). Projector (1962) estimated that an intensity of 250,000 candles was required to provide visual range of 3 miles, based on a background luminance of 1,000 foot-lamberts $\left(3,426\right.$ lumens $\left./ \mathrm{m}^{2}\right)$. Projector (1962) further added that the position of the sun further complicated visibility of aircraft lighting during daytime hours. "The sun itself has a maximum luminance about half-a-billion foot-lamberts [1.7 billion lumens $/ \mathrm{m}^{2}$ ]. The extreme difficulty of looking into the sun, or even near it, makes it generally impractical to attempt sighting in these directions" (p. 53).

\section{Strobe light evaluation}

In an evaluation of strobe lighting systems for the Department of Defense, the United States Air Force (USAF) released a report (USAF, 1977) compiling findings from a series of reports and experiments related to high-intensity flashing xenon lighting, or strobe lights. The report amassed a vast literature review and results summation of various visibility tests conducted during both daytime and nighttime conditions. While the report supported the use of strobes for select nighttime operations, the authors concluded that "strobe lights of practical intensities aren't effective in enhancing visual conspicuity of aircraft under most daylight flight conditions" (USAF, 1977, p. 133). "The analysis fails to support high intensity strobes for the reduction of daytime mid-air collision potential with present day technology" (USAF, 1977, p. 143).

Nighttime results fared significantly better, with detectability at substantially further ranges than daytime testing; however, the authors warned that the results were likely tainted by the extremely dark background luminance in the study.
As a result, the authors summarized that the collected data supported no clear conclusions and strobe lighting data were invalid at times of increased background luminance, such as during dawn or dusk. These results supported the conclusions of a similar FAA study on see and avoid, which stated "the use of anti-collision lights at night provide good conspicuity," but rejected the notion that lighting was effective during the daytime stating, "aircraft colors or lights play no significant role in first directing a pilot's attention to the 'other' aircraft during daytime" (Graham, 1989, p. 9).

\section{Light-emitting diode study}

In a recent study of aircraft anti-collision light-emitting diodes (LEDs), researchers conducted a visibility study to evaluate the accuracy of visibility prediction models based on light intensity and flashing patterns (Yakopcic et al., 2013). Conducted at the University of Dayton, the study exposed 72 participants between 18 and 25 years of age to a series of three visibility experiments. The experiments evaluated various LED flash pattern sequences at exposure intensities ranging in duration from $20 \mathrm{~ms}$ to $5 \mathrm{~s}$. While the study did not offer practical guidance regarding detection distance threshold, the authors concluded that the use of LED lighting required the creation of different visibility models to more accurately predict visibility based on LED intensity (Yakopcic et al., 2013).

Unfortunately, none of these studies addressed unmanned aircraft lighting. While the findings of the manned studies were conclusive, the authors believed that disparity between manned and unmanned aircraft operations was adequately different enough to warrant pursuit of the current study rather than accept the applicability of the manned study findings to unmanned aircraft. Nevertheless, the manned studies provided critical background and methodological considerations that played a role in this study's design and implementation.

\section{Purpose}

This research represented the second phase in a five-part series of experiments involving UAS detection, visibility, and collision avoidance. The purpose of this study was to assess the effectiveness of pilot detection of UAS platforms that were equipped with strobe lighting. Such data could be used to determine if external strobe lighting is a viable means of hastening and enhancing pilot detection and avoidance of UAS platforms to curb potential collision threats in the National Airspace System. The authors sought to determine an aware pilot's mean detection distance to strobe-lighted UAS platforms operating under daylight Visual Meteorological Conditions based on various UAS convergence conditions. The findings from this project were used to evaluate the efficacy of requiring UAS to be equipped with strobe lighting systems. The authors further aimed to develop operational recommendations to improve pilot visibility, detection, and 
collision avoidance of UAS operating at low altitude in the National Airspace System. This research could also be used to improve collision avoidance training and aeronautical decision-making for both pilots and remote pilots.

\section{Research Question}

The study sought to answer the following research question: Does platform strobe lighting significantly affect initial UAS detection range?

\section{Method}

The study used a mixed-method research design that incorporated both quantitative and qualitative components. Ten pilot-qualified participants were recruited from a Part 141 collegiate flight program in the Midwestern United States using a purposeful sampling technique. The number of participants was limited primarily by study funding restrictions.

Table 1 identifies participant pilot qualifications. To ensure both study validity and reliability, researchers intentionally selected pilots of varying degrees of experience and ability, ranging from new student pilots to seasoned flight instructors.

Table 1

Participant aeronautical demographics.

\begin{tabular}{lccc}
\hline Participant & $\begin{array}{c}\text { FAA pilot } \\
\text { certificate(s) }\end{array}$ & $\begin{array}{c}\text { Medical } \\
\text { certificate }\end{array}$ & $\begin{array}{c}\text { Reported } \\
\text { vision }\end{array}$ \\
\hline 1 & SP & 2nd Class & $20 / 25$ \\
2 & CFI & 1st Class & Unknown \\
3 & SP & 1st Class & $20 / 20$ \\
4 & CPL & 3rd Class & Unknown \\
5 & PPL & 3rd Class & Unknown \\
6 & CFII & 3rd Class & Unknown \\
7 & PPL-IR & 3rd Class & $20 / 20$ \\
8 & ATP & 1st Class & $20 / 20$ \\
9 & CFI & 1st Class & Unknown \\
10 & PPL-IR & 1st Class & $20 / 20$ \\
\hline
\end{tabular}

Note. $\mathrm{SP}=$ Student Pilot; $\mathrm{PPL}=$ Private Pilot License; $\mathrm{IR}=$ Instrument Rating; $\mathrm{CPL}=$ Commercial Pilot License; $\mathrm{CFI}=$ Certified Flight Instructor; CFII $=$ Certified Flight Instructor-Instrument; ATP $=$ Airline Transport Pilot.
Each participant's visual acuity was self-reported, and not assessed as a factor of the study. For evaluation purposes, it was assumed that participants holding either a First or Second Class Medical Certificate had an acuity of at least " $20 / 20$ or better [distant vision] in each eye separately, with or without correction" and those with a Third Class Medical Certificate had an acuity of at least "20/40 or better in each eye separately, with or without correction" (FAA, 2006).

Participants performed single-pilot duties aboard a Cessna 172 S aircraft equipped with a G-1000 avionics suite (Figure 2). The participants were given maneuver instructions that, in coordination with ground UAS pilot operators, brought them into a lateral convergence condition with an unmanned aircraft.

The experiment was conducted between July 10, 2017 and July 14, 2017 between 18:00L and 20:00L. Both manned aircraft and UAS flights were operated in accordance with operational privileges and restrictions denoted in Certificate of Authorization 2016-CSA-72-COA. Researchers flew the DJI Matrice 100 sUAS platform during the experiment (Figure 2).

The sUAS was equipped with a small array of solid and flashing LED lights to facilitate the test condition described in the research question. While aircraft lighting is not normally required for either hobbyist or commercial operations during daylight hours, the researchers wanted to evaluate the impact that such lighting would have on participant visibility.

With the exception of civil twilight operations, there are currently no regulatory standards for unmanned aircraft lighting requirements. Researchers, therefore, decided to use regulatory requirements for civil twilight operations as the basis for determining lighting requirements. According to $14 \mathrm{CFR}$ 107.29(b) provisions for civil twilight operations:

No person may operate a small unmanned aircraft system during periods of civil twilight unless the small unmanned aircraft has lighted anti-collision lighting visible for at least 3 statute miles [emphasis added].
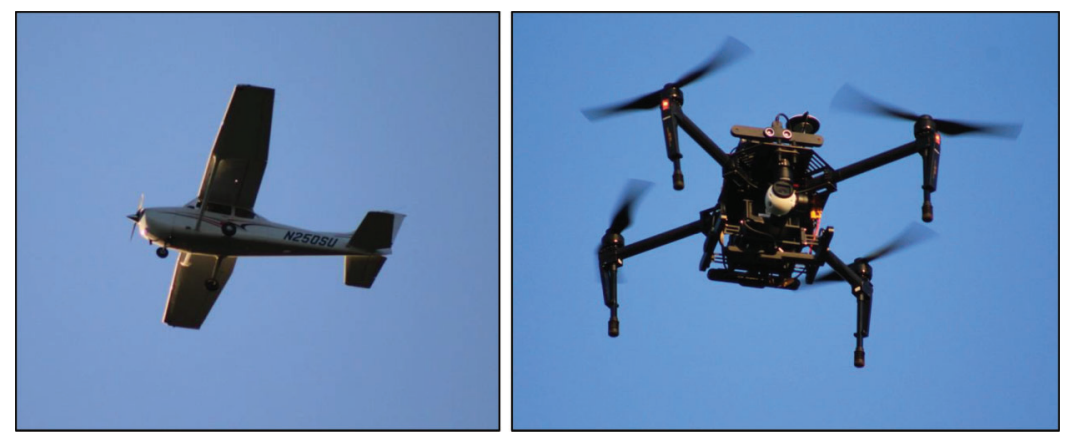

Figure 2. [Left] G-1000-equipped Cessna 172/S aircraft used during the experiment. [Right] DJI Matrice 100 outfitted with 50mAh STROBON navigation strobe lights affixed to UAS anterior surface. STROBON technical information available from: http://www.flytron.com/ led-systems/150-strobon-navigation-strobe-blue.html. 
The remote pilot in command may reduce the intensity of the anti-collision lighting if he or she determines that because of operating conditions, it would be in the interest of safety to do so. (Small Unmanned Aircraft Systems, 2017)

\section{Safety Provisions}

\section{Operations box}

The flight experiment was limited to a defined operations area in proximity to a remote control aircraft airfield. The operations box included specific navigation entry and exit points that allowed for consistent intercept geometry and timing. Moreover, the operations box confined the UAS flight area, allowing for quick aircraft escape in the event of an emergency or other unexpected flight condition aboard the manned aircraft.

\section{Certified Flight Instructor (CFI)}

A CFI occupied the co-pilot position aboard the manned aircraft during each experimental flight (Figure 3). The CFI observed for traffic conflicts, monitored aircraft radios, verified altitude and navigation compliance, and ensured overall safety of flight. Safety of flight was also assessed by an onboard test director, who assisted the CFI in coordinating with ground UAS operators.

\section{Safety altitude differential}

A small, 200-foot AGL altitude margin was built into the experiment to stimulate participant perception of an actual near-collision condition, while ensuring that both the manned aircraft and sUAS platform remained vertically de-conflicted. This margin was determined by the research team as an acceptable risk mitigation to alleviate an actual aircraft-sUAS collision. Prior to each pass, the CFI announced the aircraft's altitude on a discrete frequency to ground UAS controllers before entering the operations box. For this experiment, all UAS flights occurred below the manned aircraft, to avert the risk of a falling UAS impact.

\section{$A D S-B$ tracking}

The experimental sUAS platform was equipped with a small, proprietary ADS-B transmitter that broadcasted its position and altitude information. An ADS-B receiver device was surreptitiously monitored in the cockpit by the CFI on a tablet to ensure positive altitude de-confliction from the sUAS platform. A sample screen capture of this system is presented in Figure 4. This device was used by the onboard CFI and test director for situational awareness and safety purposes. The display was not revealed to the experiment participants, nor was it used to collect study data.

\section{Data Collection}

During the experiment the manned aircraft participants were given maneuver instructions that facilitated the following series of intercept conditions:

- Intercept 1: UAS hovered slightly left [portside] of aircraft course.

- Intercept 2: UAS hovered slightly right [starboard] of aircraft course.

- Intercept 3: UAS conducted a repeating lateral maneuver directly in front of aircraft.

- Intercept 4: UAS flew on head-on convergence course with manned aircraft.

- Intercept 5: This pass was a control pass, with no UAS airborne.

Intercepts were partially randomized to prevent interparticipant communication from compromising results. For simplification of research reporting and discussion, all intercept nomenclatures in this report were written to correspond to the appropriate intercept number and type.

The test director occupied the backseat of the aircraft during each experimental sortie. During each intercept the experiment participant would indicate sighting the UAS. The test director would record the exact time of sighting using the onboard G-1000 GPS time. Immediately following each intercept, the test director conducted a standardized verbal survey to query the experiment participant about the visual characteristics that drew their attention

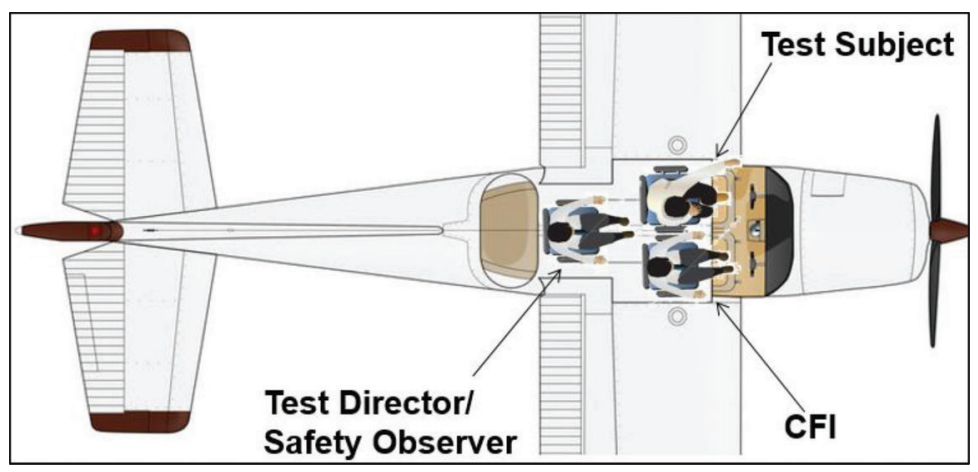

Figure 3. Aircraft experimental seating configuration. 


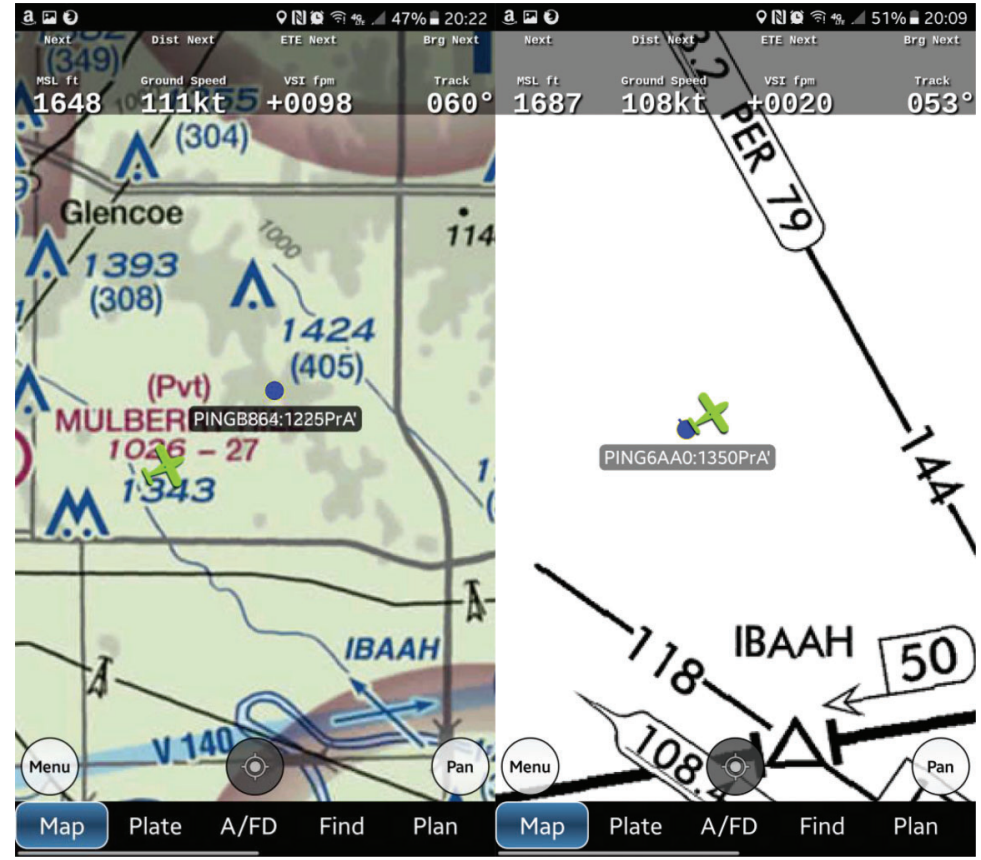

Figure 4. Small UAS ADS-B tracking data overlaid with digital [left] sectional chart and [right] IFR chart. UAS position indicated by blue marker, with altitude indicated in feet (MSL).

to the UAS and recorded the results in the research notes. The researcher also documented other relevant qualitative data or comments made by the experiment participant.

Following the experiment, researchers correlated the intercept timestamps recorded by the onboard researcher to the GPS position locations from the UAS, using recorded telemetry data. Telemetry was downloaded from the UAS into a CSV file. Similar manned aircraft telemetry and location data were extracted from the manned aircraft's G-1000 avionics suite in the same format. Collected geolocation coordinate data were compared in Excel using a coordinate distance calculator to determine the range and orientation of the UAS relative to the manned aircraft during each intercept.

\section{Assumptions}

Researchers made the following assumptions with regard to this mixed-method research:

- The skill and experience of experimental participants were relatively representative of most general aviation pilots of equivalent certificate levels.

- Participant visual acuity was presumed to conform with at least the minimum standards corresponding to their held FAA medical certificate.

- Positional and altitude data from the G-1000 and UAS telemetry devices were assumed to be accurate.

- The 50mAh STROBON navigation strobe lighting system was reported by the manufacturer to meet 14 CFR 107.29 provisions, which researchers accepted as compliant.

\section{Limitations}

The following limitations applied to this research project:

- Due to the expense associated with flying manned aircraft, the scope of the exercise was limited to 10 participants. The limited number of participants did not allow the collection of adequate data points for statistical inference or generalizability of study findings.

- Researchers did not adjust participant detection times for delays in verbalization. It was possible, but unlikely, that participants may have seen the UAS several seconds in advance of verbalizing their discovery.

- The UAS encountered a battery failure during Participant 6's fourth intercept. Researchers elected to re-accomplish the intercept.

- The airborne aircraft was forced to abort the experiment during Participant 10's fourth intercept due to high winds, rain, and nearby convective activity. Researchers did not repeat this intercept.

\section{Findings and Discussion}

The experiment was conducted on July 10-14, 2017 between 18:00 and 20:00 local time. Ten participants took part in the experiment. The experiment was conducted simultaneously with a related ground visibility research project. 
Table 2

UAS detection range by participant and intercept.

\begin{tabular}{|c|c|c|c|c|c|}
\hline Intercept & 1 & 2 & 3 & 4 & 5 \\
\hline $\begin{array}{l}\text { UAS } \\
\text { maneuver } \\
\text { aspect }\end{array}$ & $\begin{array}{c}\text { Hover } \\
\text { port }\end{array}$ & $\begin{array}{c}\text { Hover } \\
\text { starboard }\end{array}$ & $\begin{array}{c}\text { Transition } \\
\text { forward } \\
\text { lateral }\end{array}$ & $\begin{array}{l}\text { Intercept } \\
\text { head-on }\end{array}$ & $\begin{array}{l}\text { Control: } \\
\text { no sUAS }\end{array}$ \\
\hline $\begin{array}{l}\text { Participant } \\
\# 1\end{array}$ & 0.00 & \multicolumn{3}{|c|}{ Participant } & 0.00 \\
\hline$\# 2$ & 0.00 & 0.00 & 0.00 & 0.15 & 0.00 \\
\hline$\# 3$ & 0.00 & 0.00 & 0.00 & 0.00 & 0.00 \\
\hline$\# 4$ & 0.00 & 0.00 & 0.00 & 0.00 & 0.00 \\
\hline$\# 5$ & 0.00 & 1.43 & 0.00 & 0.00 & 0.00 \\
\hline \#6 & 0.00 & 0.00 & 0.00 & $0.00^{\mathrm{a}}$ & 0.00 \\
\hline$\# 7$ & 0.00 & 0.00 & 0.00 & 0.00 & 0.00 \\
\hline$\# 8$ & 0.00 & 0.00 & 0.00 & 0.00 & 0.00 \\
\hline$\# 9$ & 0.00 & 0.00 & 0.00 & 0.00 & 0.00 \\
\hline$\# 10$ & 0.00 & 0.00 & 0.00 & $\mathrm{~N} / \mathrm{A}^{\mathrm{b}}$ & 0.00 \\
\hline
\end{tabular}

Note. All distances reported in statute miles (SM). Zero values indicate no sighting.

${ }^{\mathrm{a}}$ Intercept repeated, data retained in calculations.

${ }^{\mathrm{b}}$ Intercept not repeated, data removed from calculations.

\section{Quantitative Data}

Data were successfully collected for 49 intercepts. No false sUAS detections were made during the 10 control intercepts. Participants visually detected the sUAS during $7.7 \%(n=3)$ of the 39 possible intercepts. Findings are presented in Table 2. The three sUAS detections occurred between $0.15 \mathrm{SM}$ and $2.42 \mathrm{SM}$.

This $7.7 \%$ sUAS detection rate was surprisingly low, considering the results of the Loffi et al. (2016) visibility study, which recorded a $36.8 \%$ detection rate at a mean distance of less than 0.10 SM for the Iris quadcopter. The Iris quadcopter used in the Loffi et al. (2016) study was only slightly smaller in size compared to the DJI Matrice 100 used in the current study. The Iris and DJI Matrice 100 are comparable in size, with the Iris measuring $55 \mathrm{~cm}$ motor-to-motor and the Matrice 100 measuring $65 \mathrm{~cm}$ from diagonal wheelbases (DJI, 2018; Sciautonics, 2018). The intercept conditions between the UAS and manned aircraft were similar to those the Loffi et al. (2016) study, with control group, starboard, port, transitioning, and head-on intercept conditions replicated. The only notable difference between the studies were the 2016 study's use of the fixedwing Anaconda UAS for the head-on intercept condition and an experimental execution failure which resulted in no starboard-side hovering intercepts being conducted.

Unfortunately, the low detection rate coupled with the limited number of overall data points prevented calculating meaningful statistics or making statistically based conclusions. As a result, the authors were unable to answer the primary research question.

\section{Qualitative Data}

While the availability of quantitative data was limited during this study, researchers collected a plethora of qualitative and observational data from the participants. Qualitative findings were assessed for recurring, thematic data and presented with a sampling of participant comments.

\section{Sun glare}

Sun glare presented a serious impediment to successful detection. Of the three participants who sighted the sUAS, two commented about the difficulty associated with detecting the craft when flying on westerly headings, in the direction of the sun. Participant 1 stated, "It was very difficult to see the sUAS...the afternoon sun was a factor... the afternoon sun filled the cockpit." Participant 2 similarly stated, "could not see the UAS easily, especially flying into the sun." Despite not visually detecting the sUAS, several other participants also commented about the impact of sun glare. Participant 8 stated, "it was quite difficult to see when expecting to see the UAS; the time of day with the sun had an effect-especially when facing into the sun." Participant 9 also alluded to glare issues, saying, "Difficult lighting, especially west-bound." This finding is consistent with Projector's (1962) research that suggested that daytime detectability of lighted aircraft would be extremely difficult, if not impossible, when looking into or near the sun. This may be a probable explanation for the substantial difference in detectability with the Loffi et al. (2016) study, since the prior study was conducted during peak daylight hours.

\section{UAS ground activity}

While most participants were unable to successfully detect the UAS in flight, one participant observed evidence that UAS operations were taking place, based on the ground movement of UAS operations personnel. Participant 6 commented, "I felt it was very difficult to see the $\mathrm{UAV}$, but was able to identify it on the ground after seeing the operator walk towards it." While this activity was not noted by additional participants, this observation supports the Colorado Agricultural Aviation Association's recommendations to further study the efficacy of high-visibility ground markings to highlight UAS activity locations.

\section{Strobe lighting}

None of the participants indicated definitively spotting the strobe light. Of the three participants who detected the sUAS, only Participant 4 indicated they "may have seen a blinking light," but was not confident in the observation. This observation seems to confirm that the findings of Projector (1962) and USAF (1977) — that aircraft strobe lights were relatively ineffective during daylight operations-also apply to UAS operations.

\section{Low confidence in ability to sight sUAS}

Of the 10 participants, $90 \%$ indicated that the sUAS was "difficult" or "very difficult" to see. Forty percent of participants stated they had "low" or "no" confidence in their 


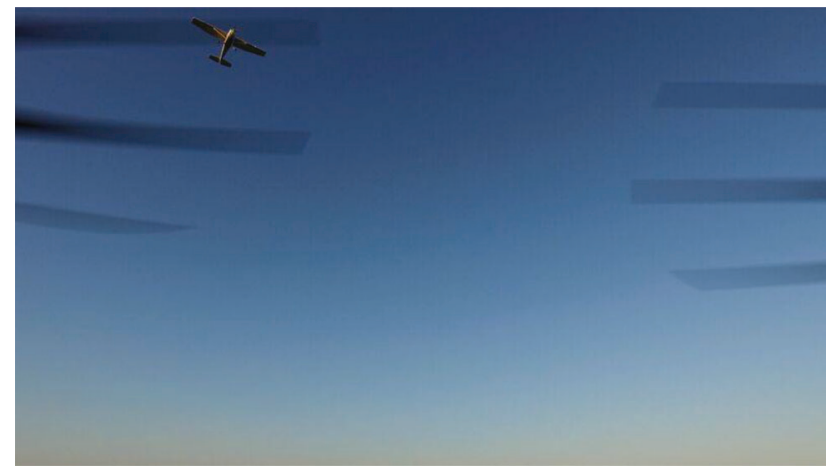

Figure 5. Reciprocal image of the manned aircraft intercepting the DJI Matrice 100 captured by the UAS's electro-optical camera.

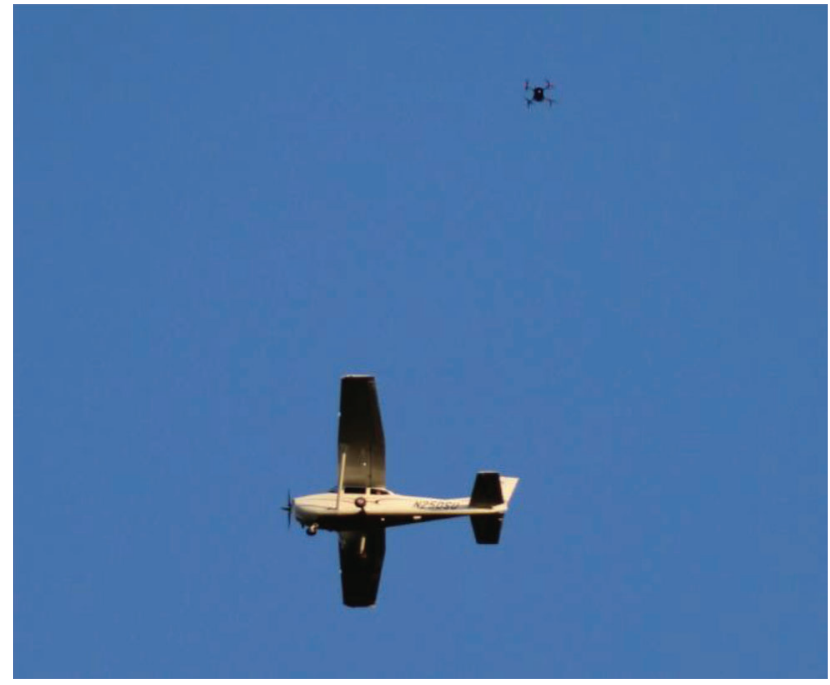

Figure 6. Ground observer image of Participant 3, Intercept 3 at time of closest approach to the sUAS. Separation between the aircraft and sUAS was 267 feet laterally and 200 feet vertically. Participant 3 did not detect the sUAS.

ability to detect the sUAS. Figures 5 and 6 illustrate the relative proximity of the UAS-aircraft intercepts from the perspective of the UAS and ground observer, respectively.

\section{Conclusions}

While it was not possible to assess the data for statistical inference, the qualitative data suggested that sUAS lighting is relatively ineffective in improving pilot sUAS detection or collision avoidance during daylight hours, further validating the conclusions of Projector (1962). The challenges associated with sun glare and high ambient lighting generally preclude this method as a means of reliable detection. This conclusion was consistent with the USAF (1977) findings that determined anti-collision lighting was ineffective during daylight hours and had questionable validity during periods of high ambient lighting, such as dawn or dusk. Further, it is possible that participant visual detection may have been compromised on up to $50 \%$ of the intercepts due to sun glare, since the manned aircraft conducted successive intercepts in reversing east-west directions. Multiple participants noted a general inability to detect the sUAS when flying westerly headings into the sun. This impediment seems to reflect the Projector (1962) study findings, in which participants reported similar challenges when flying in the general direction of the sun.

It is important to note that the experiment was conducted under highly favorable conditions. The participants flew in a high-wing Cessna aircraft, which generally maximizes look-down visibility. Additionally, the participants were made aware there would be sUAS activity in the vicinity of the operations area, which likely influenced more external traffic scanning than would normally occur during general aviation flight. Finally, the flights were conducted in relatively good weather and clear visibility conditions, thereby improving the likelihood of detection. Despite these favorable experimental conditions, UAS spotting by the participants remained exceedingly poor.

\section{Recommendations}

While the authors are not confident that lighting supports improved daylight detection, they recommend further research to explore the effectiveness of using sUAS-mounted strobe lights for nighttime visual detection.

During the course of the literature review and subsequent study, the authors also identified a unique aircraft lighting proposal to the FAA. In the document, Projector (1962) suggested adapting exterior aircraft lighting with an encoding device to transmit parametric flight information via the light's flash pattern. While these recommendations were never implemented in manned aircraft, their application when adapted to unmanned aircraft could potentially improve pilot situational awareness of UAS night operations.

Since the vast majority of existing sUAS flights occur in low-altitude airspace, the authors propose that an electronic controller could be developed to interface with the GPS receiver or other altitude source of the unmanned aircraft and code the UAS altitude into a strobe light flash pattern that represents altitude in hundreds of feet AGL. For example, four short flashes followed by a period of darkness would equate to 400 feet AGL. This approach could quickly and easily visually communicate altitude information of low-flying UAS to the remote pilot, visual observers, and proximate manned aircraft pilots. This method further alleviates manned aircraft pilots from referencing altitude information sources that are interior to the aircraft, such as UAS-adapted TCAS or ADS-B. To date, there are few-if any-existing systems for sUAS that can achieve this end. The authors are in the process of patenting this technology and plan to study the efficacy of this method in a subsequent research project. 


\section{References}

Academy of Model Aeronautics. (2016). An analysis of the FAA's March 2016 UAS sightings. Retrieved from http://amablog.modelaircraft.org/ amagov/files/2016/06/AMA-Analysis-FINAL-6-1-16.pdf

Academy of Model Aeronautics. (2017). As drone sales soar, vast majority of reports remain simple sightings. Retrieved from https://www.model aircraft.org/files/UASSightingsAnalysisbyAMA5-10-17.pdf

Bullough, J. D. (2011). Aviation signal lighting: Impacts of lighting characteristics. Advances in Applied Science Research, 2(1), 16-27. Retrieved from http://www.imedpub.com/articles/aviation-signallighting-impacts-of-lighting-characteristics-on-visibility.pdf

DJI. (2018). Matrice 100 Specs. Retrieved from https://www.dji.com/ matrice100/info

Federal Aviation Administration. (2006). Guide for aviation medical examiners: Synopsis of medical standards. Retrieved from https:// www.faa.gov/about/office_org/headquarters_offices/avs/offices/aam/ ame/guide/standards/

Federal Aviation Administration. (2016). Pilots' role in collision avoidance. Advisory Circular, AC 90-48D. Retrieved from https://www.faa. gov/documentLibrary/media/Advisory_Circular/AC_90-48D.pdf

Federal Aviation Administration. (2017). UAS sightings report [database]. Retrieved from https://www.faa.gov/uas/resources/uas_sightings_ report/

General Operating and Flight Rules. (2017). 14 CFR 91.

Gettinger, D., \& Michel, A. H. (2015). Drone sightings and close encounters: An analysis. Center for the Study of the Drone at Bard College. Retrieved from http://dronecenter.bard.edu/drone-sightings-and-close-encounters/

Graham, W. (1989). See and avoid/cockpit visibility. FAA Technical Report DOT/FAA/CT-TN/89/18. Retrieved from www.dtic.mil/get-trdoc/pdf?AD $=$ ADA2 14214

Kephart, R. J., \& Braasch, M. S. (2008). See-and-avoid comparison of performance in manned and remotely piloted aircraft. IEEE Aerospace and Electronic Systems Magazine, 25(5), 36-42. https://doi.org/ 10.1109/MAES.2010.5486540
Kochenderfer, M. J., Espindle, L. P., Kucher, J. K., \& Griffith, J. D. (2008). A comprehensive aircraft encounter model of the National Airspace System. Lincoln Laboratory Journal, 17(2), 41-53. Retrieved from https://www.1l.mit.edu/publications/journal/pdf/vol17_no2/17_2_ 2Kochenderfer.pdf

Loffi, J. M., Wallace, R. J., Jacob, J. D., \& Dunlap, J. C. (2016). Seeing the threat: Pilot visual detection of small unmanned aircraft systems in visual meteorological conditions. International Journal of Aviation, Aeronautics, and Aerospace, 3(3). Retrieved from http://commons. erau.edu/ijaaa/vol3/iss $3 / 13$

Maddocks, J., \& Griffitt, G. (2015). Qualitative evaluation of unmanned aircraft visibility during agricultural flight operations. Avian. Limited Access Report obtained from the Colorado Agricultural Aviation Association.

Michel, A. H., \& Gettinger, D. (2016). Analysis of new drone incident reports. Center for the Study of the Drone at Bard College. Retrieved from http://dronecenter.bard.edu/analysis-3-25-faa-incidents/

Moored Balloons, Kites, Amateur Rockets, Unmanned Free Balloons, and Certain Model Aircraft. (2017). 14 CFR 101.

Projector, T. H. (1962). The role of exterior lists in mid-air collision avoidance. Federal Aviation Agency Report No. FAA/BRD-127, Project No. 110-512R. Federal Aviation Agency. Retrieved from http://www.dtic. $\mathrm{mil} / \mathrm{dtic} / \mathrm{tr} / \mathrm{fulltext} / \mathrm{u} 2 / 602421 . p d f$

Sciautonics. (2018). 3D Robotics Iris plus drone review. Retrieved from http://www.sciautonics.com/3d-robotics-iris-plus-drone-review/

Small Unmanned Aircraft Systems. (2017). 14 CFR 107.

United States Air Force. (1977). ADS strobe light evaluation. Technical report ASD-TR-77-33. Retrieved from www.dtic.mil/get-tr-doc/pdf?AD= ADA045533

Yakopcic, C., Puttmann, J., Kunz, B., Ang, C., McPherson, A., Santez, D.,...Khaouley, N. (2013). Experimental effective intensity of light emitting diodes for aircraft anti-collision lighting. FAA Report DOT/ FAA/AM-13/15. Retrieved from http://www.dtic.mil/get-tr-doc/pdf? $\mathrm{AD}=\mathrm{ADA} 602270$ 